\section{"PREPRINT EXTENDED ABSTRACT" " \\ Presented Before the Division of Environmental Chemistry \\ American Chemical Society \\ San Francisco, Califomia \\ April 5-10, 1992}

\title{
Solubilization and Biodegradation of Hydrophobic Organic Compounds in Soil/Aqueous Sysiems with Nonionic Surfactants
}

\author{
David A. Edwards, Shonali Laha, Zhongbao Liu and Richard G. Luthy \\ Department of Civil Engineering \\ Carnegie Mellon University \\ Pittsburgh, PA 15213 \\ DE-FG 22-90PC 90303
}

\section{INTRODUCTION}

Nonionic surfactants may strongly interact with hydrophobic organic compounds (HOCs), soil, and microorganisms in soil/aqueous systems. These interactions affect the potential for surfactant-facilitated $\mathrm{HOC}$ transpon in soil and groundwater systems, and the feasibility of engineered surfactant cleanup of contaminated sites (McCarthy and Wober, 1991). At sufficiently high bulk liquid concentrations at $25 \mathrm{C}$, most nonionic surfactants form regular micelles in single-phase solutions, whereas certain surfactants, such as $\mathrm{C}_{12} \mathrm{E}_{4}$, may form bilayer lamellae or other types of aggregates in more complex two-phase solutions (Mitchell et al., 1983; Rosen, 1989). The critical concentrations fo: the onset of micelle and aggregate formation are termed the critical micelle concentration (CMC) and the critical aggregation concentration (CAC), respecively. Imponant changes occur in surfactant sorption, surfactant solubilization of HOCs, and microbial mineralization of HOCs in the presence of nonionic surfactants at or near these critical surfactant concentrations.

\section{EXPERIMENTAL METHODS}

Naphthalene, anthracene, phenanthrene, and pyrene are polycyclic aromatic hydrocarbons (PAHs) used as model HOC compounds. In solubilization tests with soil, batch test soil/aqueous samples with nonionic surfactant and ${ }^{14} \mathrm{C}-\mathrm{PAH}$ were rotated on a tube rotator periodically to maintain the soil in suspension during equilibration. The samples were centrifuged and aliquots were expressed through preconditioned $0.22 \mu \mathrm{m}$ ieflon filters to reduce soil-derived colloidal substances. The radioactivity was measured with a Beckman LS 5000 TD liquid scintillation counter (LSC) that employed H\# quench monitoring and automatic quench compensation technique (Liu et al., 1991a; Laha and Luthy, 1991). The extent of PAH solubilization in nonionic surfactant solution without soil was assessed for each surfactantPAH combination in aqueous batch tests as a function of surfactant dose (Edwards et al., 1991). Nonionic surfactant sorption onto soil was evaluated for each surfactant at varied sub-CMC aqueous-phase concentrations by employing a DuNuoy ring apparatus 10 measure the surface tension of bulk nonionic surfactant solutions from both aqueous and soillaqueous systems. Supra-CMC sorption of nonionic surfactant was assessed with either azo dye solubilization and spectrophotometric analysis or by measurement of chemical oxygen demand (COD), from which the amount of surfactant in bulk solution could be inferted (Liu et al., 1991b).

Aerobic microbial mineralization of phenanthrene was observed by the evolution of ${ }^{14} \mathrm{CO}_{2}$ from a soil/aqueous system comprising BOD water, soil spiked with phenanthrene solution, and a consorium of PAH-degrading bacteria that previously had been isolared from PAH-contaminated soil. The system containing the bacteria was housed in a side-arm biometer flask; the activity of the evolved ${ }^{14} \mathrm{CO}_{2}$ present in the $\mathrm{NaOH}$ was measured by LSC counting and converted to percent mineralization (Laha and Luthy, 1991).

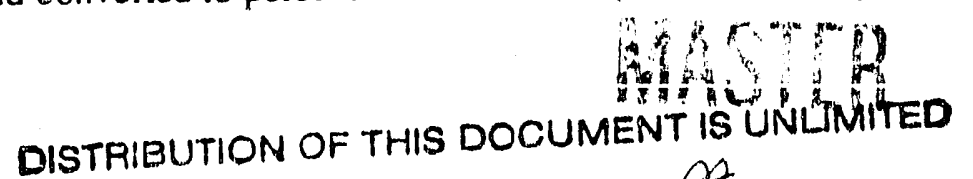




\section{Sorption of Nonionic Surfactant and HOCs onto Soil}

Sorption of nonionic surfactant onto soil diminishes the amount of surfactant available for solubilization and transport of HOC in porous soil media. Thus, the mass of nonionic surfactant that must be added before the aqueous-phase CMC is attained in a soil/aqueous system is much greater than that in an aqueous system without soil. The extent of sub-CMC sorption can be characterized with Freundlich isotherms. For the several micelle-forming nonionic surfactants and soils tested, micelles do not appear to sorb onto soil, and the amount of surfactant that sorbs thus plateaus at a maximum value at the aqueousphase CMC (Liu et al., 1991). For the lamellae-forming surfactant tested, however, maximum sorption apparently occurs at a bulk solution surfactant concentration greater than the CAC.

\section{Nonionic Surfactant Solubilization of HOCs}

In aqueous or soil/aqueous systems, hydrophobic organic compounds can be solubilized within the hydrophobic interiors of nonionic micelles, increasing the apparent bulk solution HOC solubility relative to the HOC solubility in pure water. Micellar solubilization in the bulk solution is initiated at the aqueousphase CMC or CAC. The partitioning of HOC between the micellar pseudophase and the aqueous pseudophase can be quantified with a partition coefficient, $\mathrm{K}_{\mathrm{m}}$, which is the mole fraction of HOC in the micellar pseudophase divided by the mole fraction of $\mathrm{HOC}$ in the aqueous pseudophase. HOC solubilization commences at the CMC and is linear for surfactant concentrations greater than the CMC. The slope of this relationship is the molar solubilization ratio, MSR, with units of moles of HOC solubilized per mole of micellar surfactant. A value of $\mathrm{K}_{\mathrm{m}}$, which is applicable to systems in either the presence or absence of pure-phase HOC. can be calculated for each surfactant/HOC combination by using an experimental value for the MSR (Edwards et al., 1991).

In a soil/aqueous system without separate-phase HOC, the bulk solution HOC apparent solubility in the presence of nonionic surfactant is a nonlinear function of surfactant dose. Surfactant monomers present in the aqueous pseudophase may affect the value of the HOC soil/aqueous-pseudophase partition coefficient, $\mathrm{K}_{\mathrm{d}, \mathrm{cm}}$, by enhancing the apparent HOC solubility in the aqueous pseudophase; the surfactant sorbed on soil, however, tends to increase the value of the partition coefficient by increasing the fractional organic carbon content of the soil. $\mathrm{K}_{\mathrm{d}, \mathrm{cmc}}$ can be estimated from parameters for which values are known or can be estimated. The concentration of HOC in bulk solution and the fraction of HOC solubilized from soil can be modeled without resoring to fitting coefficients by employing model parameter values, e.g., $K_{m}, K_{d, c m c}$ and the surfactant sorption coefficient, obtained from independent experiments and/or estimation techniques. The results of this modeling approach are compared with experimental data in Figure 1 (Edwards et al., $1991 \mathrm{~b}$ ) for pyrene solubilized from soil as a function of dose of $\mathrm{C}_{8} \mathrm{PE}_{9.5}$ nonionic surfactant.

\section{Nonionic Surfactant Exects on HOC Biodegradation}

In cerain laboratory tests and fermentation studies, the increase of aqueous solubilities of sparingly soluble substances by surfactant incorporation has been demonstrated to result in greater substrate bioavailability for certain compounds (e.g., Efroymson and Alexander, 1991). In a soil/aqueous system, the use of aqueous surfactant solutions may result in the transfer of HOC contaminant from the soil-sorbed phase to the micellar phase. For this reason, aqueous surfactant solutions have been suggested for use in soil bioremediation effors to increase the bioavailability of hydrophobic organic compounds. With this in mind, the effects of nonionic surfactants on the biomineralization of phenanthrene in soil/aqueous systems was investigated. Results from such mineralization tests indicate that at sub-CMC surfactant levels the degradation of phenanthrene at best proceeds as fast as in the absence of surfactant.

Figure 2 shows the mineralization of phenanthrene in a soil/aqueous system as a function of time with $v a r y i n g$ concentrations of the nonionic surfactant $C_{8} P_{9.5}$. At surfactant doses in excess of $0.05 \%(v / v)$ the 
microbial mineralization of phenanthrene is completely inhibited. This occurs at a surfactant dose of about $0.1 \%$, a dose at which micelles form in the soil/aqueous system with the ratio of soil weight to water volume of about 1:8. The surfactant dose above which microbial mineralization of phenanthrene is inhibited thus appears to be related to the CMC of the surfactant in the presence of soil. Similar results were obtained for the nonionic surfactants $\mathrm{C}_{12} \mathrm{E}_{4}$ and $\mathrm{C}_{9} \mathrm{PE}_{10.5}$.

The inhibitory effect of higher surfactant doses on phenanthrene mineralization may be attributable to various phenomena, including toxic effects, preferential use of surfactant as substrate, lowering of aqueousphae PAH concentration due to solubilization, or an interference of surfactant with microbial metabolic processes. Subsequent experiments evaluated some of these possible causes (Laha ane Luthy, 1991). Based on parallel tests with ${ }^{14} \mathrm{C}$-glucose as the substrate, and more convincingly, on results from dilution experiments where supra-CMC surfactant doses were diluted to aqueous concentrations less than CMC, it appears that the microbial inhibition observed is not a toxicity effect per se. Additional tests using various other nonionic surfactants are being employed in sub- and supra-CMC doses in ongoing tests. Preliminary results appear to negate a surfactant-specific inhibitory effect.

In conclusion, sub-CMC doses of nonionic surfactant in soil/aqueous systems in the absence of separate-phase phenanthrene do not inhibit mineralization of phenanthrene, but neither do they enhance the degradation rate. At surfactant doses in excess of the aqueous-phase CMC, the nonionic surfactants exhibit an inhibitory effect on phenanthrene mineralization. The inhibition may be a result of a physicochemical effect of the surfactant micelles interfering with substrate transpor into the cell, or with the activity of enzymes and other membrane proteins of the cell.

\section{SUMMARY}

Ongoing research is investigating mechanisms of nonionic surfactant sorption onto soil, solubilization of hydrophobic organic compounds (HOCs) from soil, and microbial degradation of HOCs in soil/aqueous systems with nonionic surfactants. The solubilization of HOC from soil can be modeled using parameters obtained from independent experiments. The microbial degradation of phenanthrene in soil/aqueous systems is inhibited by addition of alkylethoxylate or alkyphenylethoxylate surfactants at doses which result in solubilization of phenanthrene from soil. Available data suggest that the supra-CMC inhibitory effect on biodegradation is not a specific toxic effect, or a consequence of the surfactant being used as a preferential substrate.

The effects of surfactant solubilization on biodegradation of HOCs need to be explored from both physicochemical and microbial perspectives. The effects of surfactant monomers and micelles on microbial cell surfaces and constituents must be better understood in order to evaluate whether synthetic surfactants may be employed advantageously to speed up rates of bioremediation. There is a need to know more of the physical and biochemical means by which surfactant may effect the transport of HOCs 10 and across cell membranes. Clearly these various questions are difficult to address, requiring combined efforts of soil scientists, environmental engineers, and microbial ecologists.

\section{REFERENCES}

Edwards, D.A., Luthy, R.G. and Liu, Z. (1991a). Solubilization of polycyclic aromatic hydrocarbons in micellar nonionic surfactant solutions. Environ. Sci. Technol 25, 127-133.

Edwards, D.A., Liu, Z., and Luthy, R.G. (1992a). Nonionic surfactant solubilization of hydrophobic organic compounds in soilaqueous systems. Submitted to J. Environ. Engr., ASCE.

Edwards, D.A., Liu, Z., and Luthy, R.G. (1992b). Comparison of experimental data with model results for nonionic surfactant solubilization of polycyclic aromatic hydrocarbons in soil/aqueous systems. Subminted to J. Environ. Engr.. ASCE.

Efroymson, R.A., and Alexander, M. (1991). Biodegradarion by an athrobacter species of hydrocarbons partitioned into an organic solvent. Appl. Environ. Microbiol. 57, 1441-1447.

Laha, S. and Luthy, R.G. (1991). Inhibition of phenanthrene mineralization by nonionic surfactants in suil-wäier sysiems. Environa. Sci. Technól., 25, 19201930. 
Liu, Z., Laha, S. and Luthy, R.G. (1991a). Surfacatant solubilization of polycyclic aromatic hydrocarbons in soil/water suspensions. Water Sci. Tech., 23, 475-485.

Liu, Z., Edwards, D.A. and Luthy, R.G. (1991b). Sorption of nonionic surfactants onto soil. Submitted to Wat. Res..

McCarthy, J.F. and Wober, F.J. (1991). Summary Report: Concepts in Manipulation of Groundwater Colloids for Environmental Restoration, Manteo, NC October 15-18, 1990, U.S. Deparment of Energy.

Mitchell, D.J., Tiddy, G.J.T., Waring, L., Bostock, T., and McDonald, M.P. (1983). Phase behaviour of polyoxyethylene surfactants with water. J. Chem. Soc., Faraday Trans. 1, 79, 975-1000.

Rosen, M.J. (1989). Surfactants and interfacial phenomena, 2nd ed., John Wiley and Sons, New York.
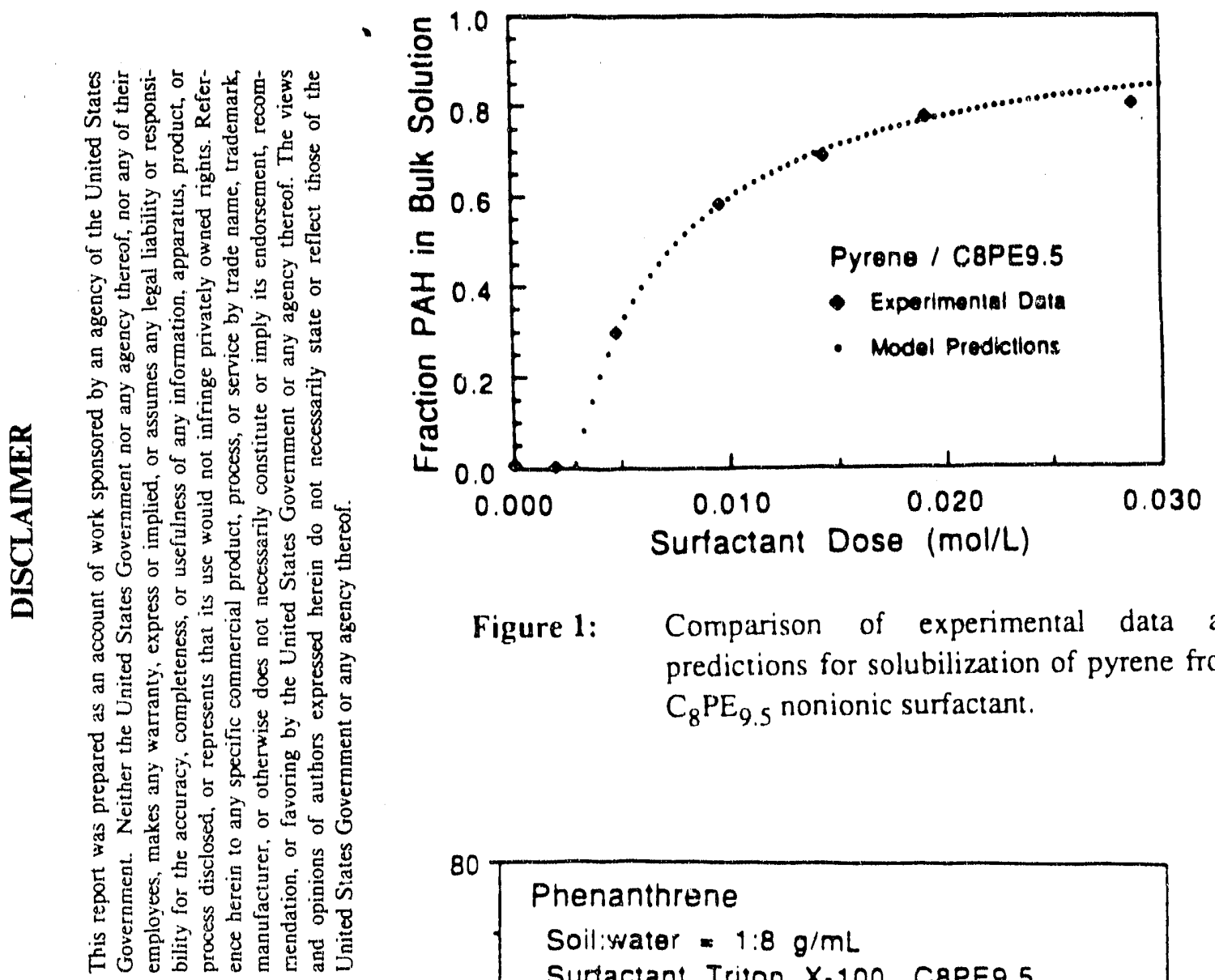

Figure 1: Comparison of experimental data and model predictions for solubilization of pyrene from soil with $\mathrm{C}_{8} \mathrm{PE}_{9.5}$ nonionic surfactant.

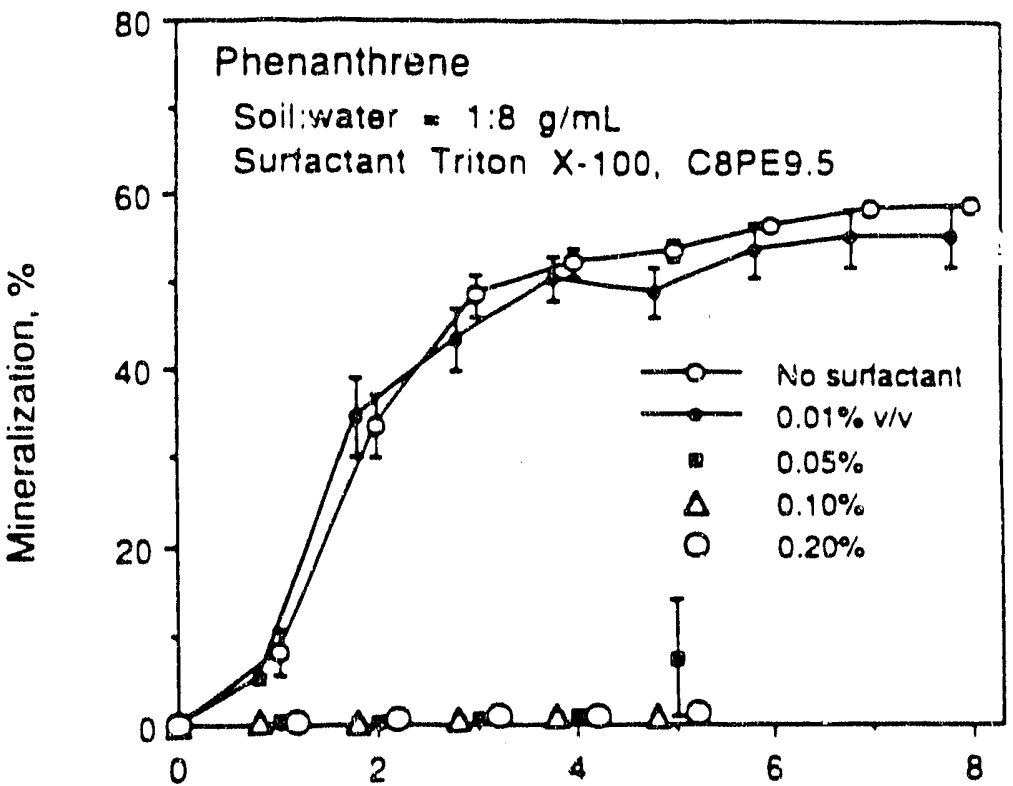

Time, weeks

Figure 2: $\quad$ Mineralization of phenanthrene in a soil/aqueous system with varying concentrations of $\overline{\mathrm{C}}_{8} \mathrm{PE}_{9.5}$ nonionic surfactant. 


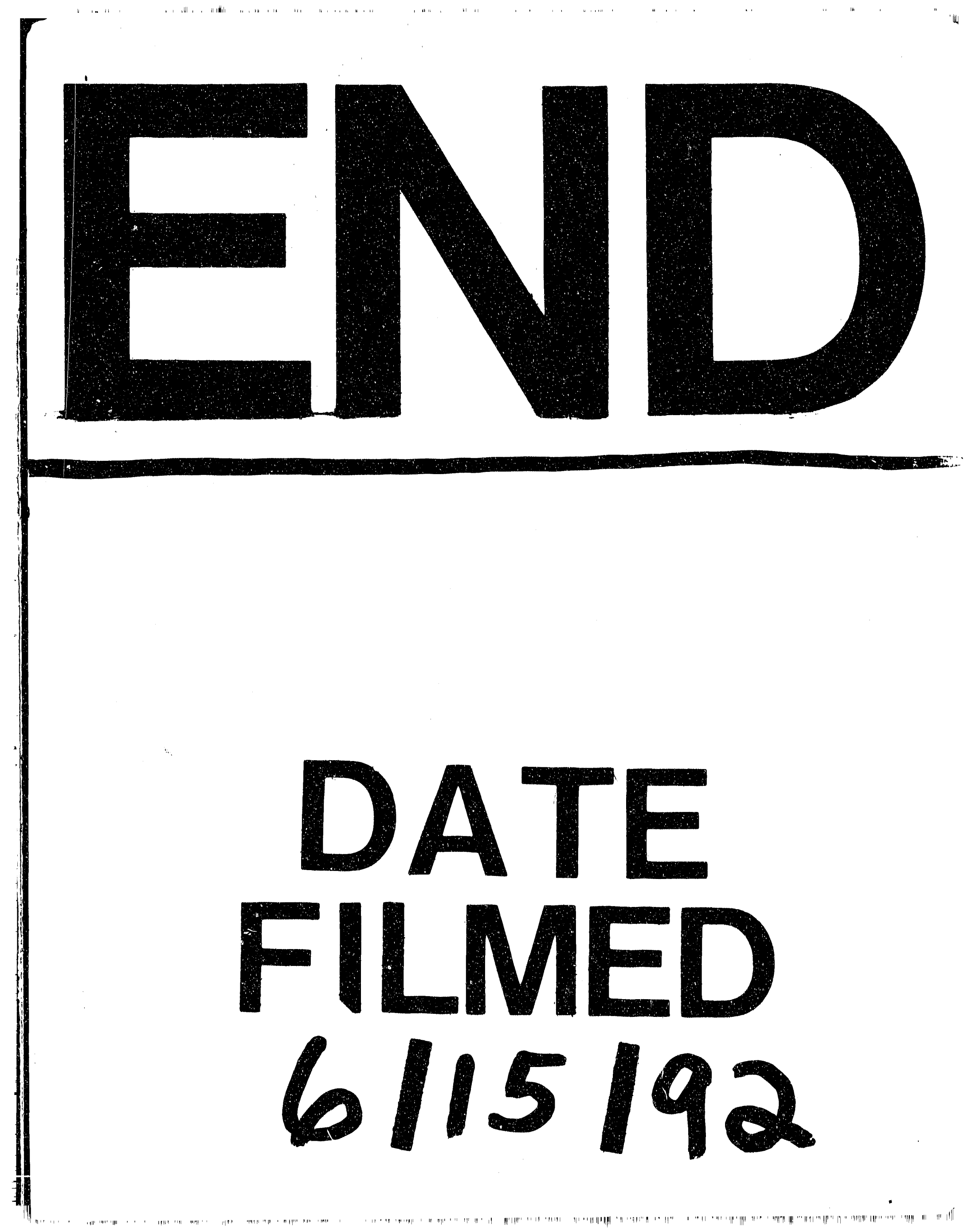


